\title{
Correction to: Evolutionarily novel genes are expressed in transgenic fish tumors and their orthologs are involved in development of progressive traits in humans
}

\author{
E. A. Matyunina ${ }^{1,2}$, A. V. Emelyanov ${ }^{3,4}$, T. V. Kurbatova ${ }^{1,2,3}$, A. A. Makashov' ${ }^{1,2,3}$, I. V. Mizgirev ${ }^{5}$ and A. P. Kozlov ${ }^{1,2,3,6^{*}}$
}

\section{Correction to: Infect Agents Cancer (2019) 14:46 https://doi.org/10.1186/s13027-019-0262-5}

The original publication of this article [1] contained 4 errors in column 1 of Table 4. In this correction article the errors and updated table are published.

Errors in original publication:

1. "Fish notch1b/Human NOTCH2" should be "Fish reck/Human RECK

2. "Fish notch1b/Human NOTCH3" should be "Fish srd5a1/Human SRD5A1

3. "Fish notch1b/Human NOTCH4" should be "Fish wnt7bb/ HumanWNT7B"

4. "Fish notch1b/Human NOTCH5" should be "Fish pparg/Human PPARG"

\section{Author details}

${ }^{1}$ Research Institute of Ultra-Pure Biologicals, Ministry of Public Health of the Russian Federation, St.-Petersburg, Russia. ${ }^{2}$ Peter the Great Saint-Petersburg Polytechnic University (SPbPU), St.-Petersburg, Russia. ${ }^{3}$ The Biomedical Center (BMC), St.-Petersburg, Russia. ${ }^{4}$ Institute for Research on Cancer and Aging (IRCAN), Nice, France. ${ }^{5}$ Petrov Research Institute of Oncology, St.-Petersburg, Russia. ${ }^{6}$ Vavilov Institute of General Genetics, Russian Academy of Sciences, Moscow, Russia.
Published online: 27 January 2020

\section{Reference}

1. Matyunina EA, Emelyanov AV, Kurbatova TV, et al. Evolutionarily novel genes are expressed in transgenic fish tumors and their orthologs are involved in development of progressive traits in humans. Infect Agents Cancer. 2019;14: 46. https://doi.org/10.1186/s13027-019-0262-5.

The original article can be found online at https://doi.org/10.1186/s13027019-0262-5

* Correspondence: contact@biomed.spb.ru

${ }^{1}$ Research Institute of Ultra-Pure Biologicals, Ministry of Public Health of the Russian Federation, St.-Petersburg, Russia

${ }^{2}$ Peter the Great Saint-Petersburg Polytechnic University (SPbPU),

St.-Petersburg, Russia

Full list of author information is available at the end of the article

(c) The Author(s). 2020 Open Access This article is distributed under the terms of the Creative Commons Attribution 4.0 International License (http://creativecommons.org/licenses/by/4.0/), which permits unrestricted use, distribution, and reproduction in any medium, provided you give appropriate credit to the original author(s) and the source, provide a link to the Creative Commons license, and indicate if changes were made. The Creative Commons Public Domain Dedication waiver (http://creativecommons.org/publicdomain/zero/1.0/) applies to the data made available in this article, unless otherwise stated. 
Table 4 Additional human orthologs of fish $\Pi_{\text {Rgr }} E E N$ genes, according to OMA ortholog search algorithm, with functions that do not exist in fish

\begin{tabular}{|c|c|c|c|c|}
\hline \multirow{2}{*}{$\begin{array}{l}\text { Name of gene } \\
\text { (Fish gene / } \\
\text { Human gene) }\end{array}$} & \multicolumn{3}{|l|}{ GO domain } & \multirow{2}{*}{$\begin{array}{l}\text { Selected GO progressive functions not encountered in fish } \\
\text { ([Fish gene] / [Human gene]) }\end{array}$} \\
\hline & $\begin{array}{l}\text { Molecular function } \\
\text { (Fish gene / Human } \\
\text { gene) }\end{array}$ & $\begin{array}{l}\text { Cellular component } \\
\text { (Fish gene / Human } \\
\text { gene) }\end{array}$ & $\begin{array}{l}\text { Biological process } \\
\text { (Fish gene / Human } \\
\text { gene) }\end{array}$ & \\
\hline $\begin{array}{l}\text { Fish atxn1l / } \\
\text { Human } \\
\text { ATXN1L }\end{array}$ & $1 / 3$ & $1 / 5$ & $0 / 10$ & [NO] / lung alveolus development \\
\hline $\begin{array}{l}\text { Fish idza / } \\
\text { Human ID2 }\end{array}$ & $1 / 3$ & $2 / 4$ & $8 / 56$ & $\begin{array}{l}\text { [NO] / epithelial cell differentiation involved in mammary gland } \\
\text { alveolus development, mammary gland epithelial cell } \\
\text { proliferation, mammary gland alveolus development, ventricular } \\
\text { septum development }\end{array}$ \\
\hline $\begin{array}{l}\text { Fish } c c r 11.1 / \\
\text { Human } \\
\text { CX3CR1 }\end{array}$ & $3 / 4$ & $2 / 7$ & $4 / 17$ & [NO] / cerebral cortex cell migration \\
\hline $\begin{array}{l}\text { HFish } \\
\text { cntnap2a } \\
\text { /Human CNTN } \\
\text { AP2 }\end{array}$ & $0 / 2$ & $2 / 15$ & $1 / 8$ & [NO] / cerebral cortex development \\
\hline $\begin{array}{l}\text { Fish mycn / } \\
\text { Human MYCN }\end{array}$ & $2 / 7$ & $1 / 3$ & $1 / 20$ & [NO] / lung development \\
\hline $\begin{array}{l}\text { Fish neflb / } \\
\text { Human NEFL }\end{array}$ & $1 / 10$ & $2 / 10$ & $1 / 29$ & [NO] / cerebral cortex development \\
\hline $\begin{array}{l}\text { Fish notchib } \\
\text { /Human } \\
\text { NOTCH1 }\end{array}$ & $3 / 15$ & $1 / 20$ & $15 / 162$ & [NO] / lung development \\
\hline $\begin{array}{l}\text { Fish reck / } \\
\text { Human RECK }\end{array}$ & $0 / 5$ & $0 / 4$ & $7 / 8$ & [NO] / embryo implantation \\
\hline $\begin{array}{l}\text { Fish srd5a1 / } \\
\text { Human } \\
\text { SRD5A1 }\end{array}$ & $2 / 7$ & $2 / 11$ & $4 / 40$ & [NO] / cerebral cortex development \\
\hline $\begin{array}{l}\text { Fish wnt7bb / } \\
\text { Human WNT7B }\end{array}$ & $2 / 3$ & $3 / 9$ & $4 / 42$ & $\begin{array}{l}\text { [NO] / trachea cartilage morphogenesis, lobar bronchus } \\
\text { development, lung epithelium development, lung development, } \\
\text { lung morphogenesis, chorio-allantoic fusion, embryonic placenta } \\
\text { morphogenesis, mammary gland epithelium development }\end{array}$ \\
\hline $\begin{array}{l}\text { Fish pparg / } \\
\text { Human PPARG }\end{array}$ & $7 / 30$ & $2 / 8$ & $6 / 81$ & [NO] / placenta development \\
\hline
\end{tabular}

